\title{
Corrections to the neutrinoless double-beta-decay operator in the shell model
}

\author{
Jonathan Engel \\ Dept. of Physics and Astronomy, University of North Carolina, Chapel Hill, NC, 27516-3255, USA \\ Gaute Hagen \\ Physics Division, Oak Ridge National Laboratory, P.O. Box 2008, Oak Ridge, TN 37831, USA.
}

\begin{abstract}
We use diagrammatic perturbation theory to construct an effective shell-model operator for the neutrinoless double-beta decay of ${ }^{82} \mathrm{Se}$. The starting point is the same Bonn-C nucleon-nucleon interaction that is used to generate the Hamiltonian for recent shell-model calculations of double-beta decay. After first summing high-energy ladder diagrams that account for short-range correlations and then adding diagrams of low order in the $G$ matrix to account for longer-range correlations, we fold the two-body matrix elements of the resulting effective operator with transition densities from the recent shell-model calculation to obtain the overall nuclear matrix element that governs the decay. Although the high-energy ladder diagrams suppress this matrix element at very short distances as expected, they enhance it at distances between one and two fermis, so that their overall effect is small. The corrections due to longer-range physics are large, but cancel one another so that the fully corrected matrix element is comparable to that produced by the bare operator. This cancellation between large and physically distinct low-order terms indicates the importance of a reliable nonperturbative calculation.
\end{abstract}

\section{INTRODUCTION}

Neutrinoless double-beta decay is a very slow leptonnumber-violating nuclear process that occurs if neutrinos are their own antiparticles. An initial nucleus $(Z, A)$, with proton number $Z$ and total nucleon number $A$, decays to $(Z+2, A)$, emitting two electrons in the process [1].

The neutrino masses and mixing matrix figure prominently in the decay. The rate, assuming that the process is mediated by the exchange of a light virtual neutrino, is

$$
\left[T_{1 / 2}^{0 \nu}\right]^{-1}=G_{0 \nu}(Q, Z)\left|M_{0 \nu}\right|^{2}\left\langle m_{\beta \beta}\right\rangle^{2},
$$

where $Q$ is the energy difference between the initial and final nuclei, $Z$ is the charge of the initial nucleus, $G_{0 \nu}(Q, Z)$ is a tabulated phase-space measure, $M_{0 \nu}$ is the nuclear matrix element to which we turn shortly, and $m_{\beta \beta}$ is a linear combination of neutrino masses:

$$
m_{\beta \beta} \equiv\left|\sum_{k=1}^{3} m_{k} U_{e k}^{2}\right| .
$$

In this last equation, $m_{k}$ is the mass of the $k^{\text {th }}$ neutrino (these mass eigenstates are linear combinations of the electron-, mu- and tau-neutrinos) and $U_{e k}$ is the element of the unitary mixing matrix that connects that neutrino to the electron neutrino. The quantity $m_{\beta \beta}$ is what experimenters want to extract from the measured decay rate. They cannot do so, however, without knowing the matrix element $M_{0 \nu}$, which must be calculated in some nuclear model.

Most calculations of $M_{0 \nu}$ are done either in the neutron-proton Quasiparticle Random Phase Approximation (QRPA) or in the shell model. The two methods have complementary virtues. The QRPA includes many single-particle orbitals outside a relatively small "inert" core, but limits itself to a particular kind of correlation. The shell model includes arbitrary complicated correlations, but only among a few single-particle orbitals outside a larger inert core. The current predictions of the two models, after a recent shaking out period, show the QRPA matrix element exceeding that of the shell model by factors of up to about two in the lighter isotopes such as ${ }^{76} \mathrm{Ge}$ and ${ }^{82} \mathrm{Se}$, and somewhat less in the heavier isotopes [2, 3].

Which kind of calculation is closer to the truth? Are there important effects that escapes both models? To find out, one has to correct one or both to account for omitted physics. Although it is possible to add missing correlations to the QRPA, it is not easy to do so systematically because several different uncontrolled approximations - BCS pairing, a phenomenological interaction, the quasiboson approximation, etc. - are part of the method. By contrast, because the shell model includes all correlations within a well-defined subspace of the full Hilbert space (the space generated by valence particles occupying a few single-particle states), there is a systematic procedure for adding the effects of states outside that space [4, 5].

While the procedure as usually implemented is perturbative in a renormalized residual nuclear interaction (the $G$ matrix) and not always reliable for that reason, it often works well enough, particularly if followed by some modest adjustment to data. Practitioners have long used such an approach to obtain good effective interactions [6], but have never applied the same techniques to obtain an effective double-beta-decay operator. Instead, they typically modify the bare operator phenomenologically, e.g. through the reduction of the axial-vector coupling constant $g_{A}$ (suggested by studies of single-beta decay) or the use of a prescription [7] to treat the short-range nucleonnucleon repulsion that is not present in shell-model wave 
functions ${ }^{1}$. Not surprisingly, it is difficult to assess the reliability of such approximations.

In this paper, therefore, we apply the same techniques used to construct effective shell-model interactions to the decay operator itself. Section II below contains a brief description of the matrix element we try to calculate, and Section III a description of our procedure for renormalizing the corresponding operator. In Section IV we present the results of our calculation, which combines the renormalized operator with shell-model transition densities from the authors of Ref. [10] for the decay of ${ }^{82} \mathrm{Se}$ to ${ }^{82} \mathrm{Kr}$. (Densities for other decays, e.g. of ${ }^{76} \mathrm{Ge}$, are not currently available.) Although we cannot be fully confident in our perturbative result, our initial steps accounting for short-range correlations through the generation of a $G$ matrix and an analogous corrected decay operator - are nonperturbative and trustworthy. And even if the low-order perturbation theory we employ subsequently is not accurate, it should tell use whether we might expect significant renormalization in a fully nonperturbative treatment.

\section{FORM OF BARE $M_{0 \nu}$}

A precise expression for the matrix element is complicated, but with a few approximations that induce an error of with an error of less than $30 \%$ [2, 3, we can write $M_{0 \nu}$ as

$$
M_{0 \nu} \approx M_{0 \nu}^{G T}-\frac{g_{V}^{2}}{g_{A}^{2}} M_{0 \nu}^{F}
$$

with $g_{V}$ and $g_{A}$ the vector and axial-vector coupling constants, and

$$
\begin{aligned}
M_{0 \nu}^{F} & =\left\langle f\left|\sum_{a, b} H\left(r_{a b}, \bar{E}\right) \tau_{a}^{+} \tau_{b}^{+}\right| i\right\rangle, \\
M_{0 \nu}^{G T} & =\left\langle f\left|\sum_{a, b} H\left(r_{a b}, \bar{E}\right) \vec{\sigma}_{a} \cdot \vec{\sigma}_{b} \tau_{a}^{+} \tau_{b}^{+}\right| i\right\rangle .
\end{aligned}
$$

Here $|i\rangle$ and $|f\rangle$ are the initial and final nuclear ground states, $a, b$ label nucleons, $\bar{E}$ is an average excitation energy, and $H$ is a "neutrino potential", given by

$$
H(r, \bar{E})=\frac{2 R}{\pi r} \int_{0}^{\infty} d q \frac{\sin q r}{q+\bar{E}-\left(E_{i}+E_{f}\right) / 2} .
$$

The quantity $R$ is the nuclear radius, inserted to make the matrix element dimensionless. Since our work is exploratory, we use the relatively simple forms in Eqs. (4) and (5) in most of what follows, though we also discuss corrections due to nucleon form factors and forbidden terms in the weak nuclear current.

\footnotetext{
1 The use of the Unitary Correlation Operator Method, e.g, in Ref. 8, 9], is more than a prescription, but the method has not yet been consistently applied to both the decay operator and the interaction.
}

\section{CONSTRUCTING AN EFFECTIVE SHELL-MODEL DECAY OPERATOR}

\section{A. Formalism and diagrams for two-body operators}

Diagrammatic effective-operator theory has a long history in nuclear physics. References [1] and 4] are early reviews and Ref. [5] is a more recent one. The theory is based on the division of the many-body Hilbert space into the shell-model space $P$ of particles occupying several degenerate or quasidegenerate orbitals (usually eigenstates a harmonic-oscillator potential $U$ ), and the rest of the Hilbert space $Q$. One begins by defining operators with the same names that project onto these spaces:

$$
P=\sum_{i \in P}|i\rangle\left\langle i\left|, \quad Q=\sum_{\text {other } i}\right| i\right\rangle\langle i|,
$$

with

$$
P^{2}=P, \quad Q^{2}=Q, \quad P Q=Q P=0 .
$$

Next one defines an effective Hamiltonian that when acting on the $P$-space projection of an eigenstate $\left|\Psi_{a}\right\rangle$ gives back that projection with the correct eigenvalue:

$$
H_{\mathrm{eff}}\left(E_{a}\right) P\left|\Psi_{a}\right\rangle=E_{a} P\left|\Psi_{a}\right\rangle .
$$

Similarly, for any "bare" operator $\mathcal{M}$ (where "bare" means "acting in the full model space"), we can define an effective operator $\mathcal{M}_{\text {eff }}$ that acts only in the $P$ space, with matrix elements related to those of the bare operator by:

$$
\frac{\left\langle\Psi_{a}\left|P \mathcal{M}_{\mathrm{eff}} P\right| \Psi_{b}\right\rangle}{\sqrt{\left\langle\Psi_{a}|P| \Psi_{a}\right\rangle\left\langle\Psi_{b}|P| \Psi_{b}\right\rangle}}=\left\langle\Psi_{a}|\mathcal{M}| \Psi_{b}\right\rangle
$$

It is straightforward to show that the effective Hamiltonian can be represented in energy-dependent form as a solution to the Bloch-Horowitz equation 12 .

$$
H_{\mathrm{eff}}(E)=P H P+P H Q \frac{1}{E-Q H} Q H P,
$$

with the full wave function a solution to the associated equation

$$
|\Psi(E)\rangle=\mathcal{Z}\left(1+\frac{1}{E-Q H} Q H\right) P|\Psi(E)\rangle,
$$

Here $E$ is the energy of the eigenstate and $\mathcal{Z}$ is a normalization factor. One can remove the explicit dependence on energy by treating the residual interaction $V-U$, which couples the $P$ and $Q$ spaces, as a perturbation. When Eqs. (11) and (12) are solved order by order, the result is a series of valence-linked Goldstone diagrams for the matrix elements matrix elements of $H_{\text {eff }}$ and $\mathcal{M}_{\text {eff }}$ 11.

The diagrams are very much like those for the binding energy, but have open lines at each end to represent the 
valence single-particle states on the right and left sides of the effective operators. The use of this representation in a linked-cluster expansion forces the introduction of "folded" diagrams, in which intermediate states have zero excitation energy. The mostly low-order diagrams we consider here, however, will not have folds.

Some recent work on effective interactions [13] has used $V_{\text {low k }}$ as a starting point, with high-energy states effectively integrated out at the beginning. But because we want to calculate the contributions of such states to the effective decay operator, we need a method that treats them explicitly. Our starting point, therefore, is the same as in the traditional treatment of nuclear matter: we define a nonperturbative $G$ matrix as the sum of the twoparticle ladder diagrams displayed in Fig. 1 below. The ruled lines in the figure indicate high-energy states, lying well above the shell-model single-particle space (we will vary the exact amount by which they are above). The $G$ matrix is thus defined not only between two-body states in the valence space but also between states in a larger model space that contains several higher shells. (See Ref. [5] for details on this "double partitioning" of the Hilbert space.)

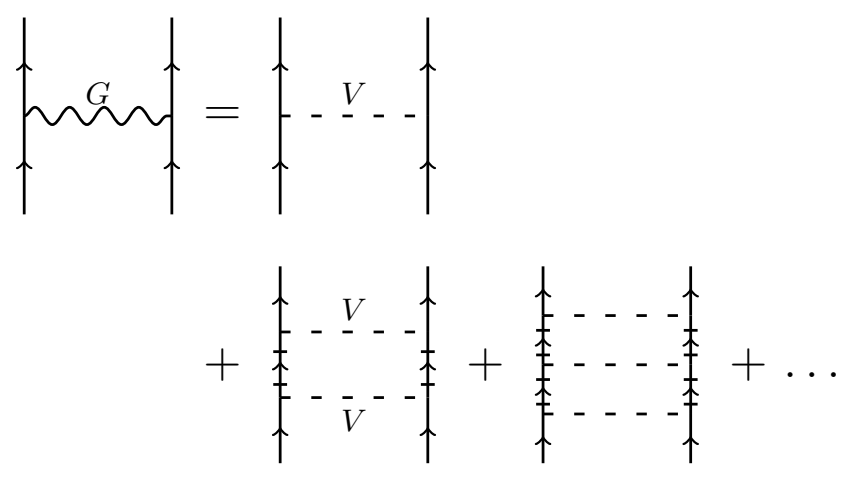

FIG. 1: Diagrammatic construction of a $G$ matrix, the first step in an effective interaction. Ruled lines correspond to high-energy particle states in the doubly partitioned space.

Although there are an infinite number of ladder diagrams, the sum can be carried out indirectly, e.g. through the solution of the Bethe-Goldstone equation. The familiar idea underlying the infinite sum is that the hard short-range core that makes the nucleon-nucleon interaction intractable can be treated exactly, at least at the two-body level, by nonperturbatively admixing into the wave function intermediate two-particle states with arbitrarily high-energy. The effective low-energy interaction $G[V]$ that results has a soft core because the effects of short-range physics have already been accounted for in the ladder sum. The argument $V$ in the $G$ matrix is meant to reinforce the fact that $G$ depends on the "bare interaction" $V$.

After this nonperturbative construction of the $G$ matrix, one can use perturbation theory in $G$ to add the effects of states that are at low energy but still outside the valence space, i.e. in the intermediate space of the
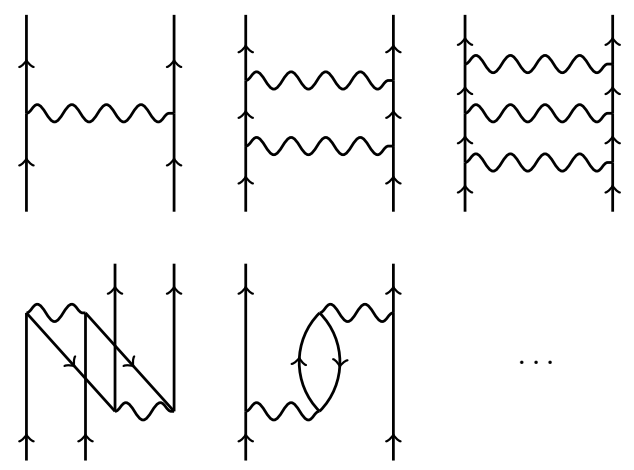

FIG. 2: Low-order diagrams (in $G$ ) for the effective interaction $H_{\text {eff }}$.

double-partitioned set. The diagrams in Fig. 2 include all such effects up to second order in $G$ (with the exception of those produced by tadpole and one-body graphs, which are commonly omitted), and some third-order effects. In this figure, the upward-going lines represent low-lying particle states (including the valence levels, as long as they do not lead to intermediate denominators with zero energy) in the gap between the Fermi surface and the high-energy levels. Downward going lines represent "hole" states that correspond to the vacating of levels below the shell-model space.

Typically, more complicated graphs, including the folded ones, are included alongside the graphs in the figure. Even then, problems with convergence, three- and higher-body operators that are generally too complicated to include, etc., mean that the resulting interaction often must be modified phenomenologically, especially in the monopole-monopole channel [6, 14]. Sometimes, however, the perturbation theory by itself is enough to produce a pretty good interaction [5, 13. The recent shellmodel calculations of double-beta decay in Ref. [10] were based on a tuned version of such an interaction.

A similar procedure can be followed to evaluate the matrix elements of any two-body operator ${ }^{2} \mathcal{M}$. The expansion of equation (12) leads to a set of diagrams for the effective operator in Eq. 10 in which a horizontal line representing the bare operator appears once alongside an arbitrary number of interaction lines [4, 11]. The denominator of Eq. (10) gives rise, in addition, to norm and overlap diagrams that determine a special basis in which the effective operator should be represented. We have, however, evaluated the most important of these diagrams (they are given by the derivative with respect to the unperturbed energy of the effective-interaction diagrams) and found them to contribute at most a few percent. We therefore won't include their complicated but small effects here.

\footnotetext{
2 Previous work has focused almost entirely on one-body operators, however.
} 
To obtain the effective decay operator, we begin by summing all diagrams with two particles excited to high energies in which one horizontal line in each diagram is the operator $\mathcal{M}$ rather than the interaction. We denote the result of this nonperturbative sum, which is completely analogous to the $G$ matrix, by $\mathcal{M}_{\text {high }}$. Sequences of $1,2, \ldots$ interaction lines either before or after the operator insertion can be separately summed, i.e. replaced by $G$ matrices (or, more, precisely, the similar ladder sum $\tilde{G}$ for which the outgoing states are high-lying). If $\mathcal{M}$ is one of the double-beta operators appearing in equations (3), then two neutron lines become proton lines whenever it acts, and the ladder sum reduces to the four diagrams in Fig. 3. Thus, the solid lines (red online) in the figure represent neutrons and the dotted lines (blue online) represent protons.

Since these diagrams involve only $T=1$ states, the their sum (with small Coulomb effects neglected) can be calculated simply from a $G$-matrix code through the trick

$$
\mathcal{M}_{\text {high }}=\left.\frac{d}{d \lambda} G\left[V_{T=1}+\lambda \mathcal{M}^{\prime}\right]\right|_{\lambda=0},
$$

where $\mathcal{M}^{\prime}$ is the charge-conserving version of the chargechanging operator in Eqs. (4) or (5), obtained by removing the isospin raising operators. In other words, we can calculate matrix elements of $\mathcal{M}_{\text {high }}$ by computing the $G$ matrix corresponding to the interaction $V+\lambda \mathcal{M}^{\prime}$. The derivative filters out all graphs except for those that have a single double-beta line replacing an interaction line. The difference between the matrix elements of $\mathcal{M}$ and $\mathcal{M}_{\text {high }}$ give us a rigorous measure, at least for twovalence-nucleon systems, of the effects of short-range correlations in double-beta decay (up to the few percent due to norm diagrams).

Having constructed $\mathcal{M}_{\text {high }}$ to include short-range twobody correlations, one can use it together with the $G$ matrix to calculate the additional renormalization from lowlying excitations. We do so by replacing one $G$-matrix line in each of the diagrams in Fig. 2 by $\mathcal{M}_{\text {high }}$. The resulting diagrams for $\mathcal{M}_{\text {eff }}$, all first order in $G$ except for second-order ladders, appear in Fig. 4 . Short-range correlations are included at every vertex through the use of $G$ and $\mathcal{M}_{\text {high }}$ in place of $V$ and $\mathcal{M}$. Most of these diagrams can be calculated through the trick in equation (13). Only the core-polarization graphs (the last two in the figure) must be treated explicitly. Those graphs are essentially different from the corresponding effectiveinteraction graph in the identities (neutron or proton) of the particles and holes involved. One cannot see the difference explicitly in our figures because Fig. 2 does not distinguish between neutrons and protons.

\section{B. Using the operator in ${ }^{82} \mathrm{Se}$}

The shell-model calculations of Ref. [10] used an interaction that was constructed largely through the effectiveinteraction theory discussed above (though it included

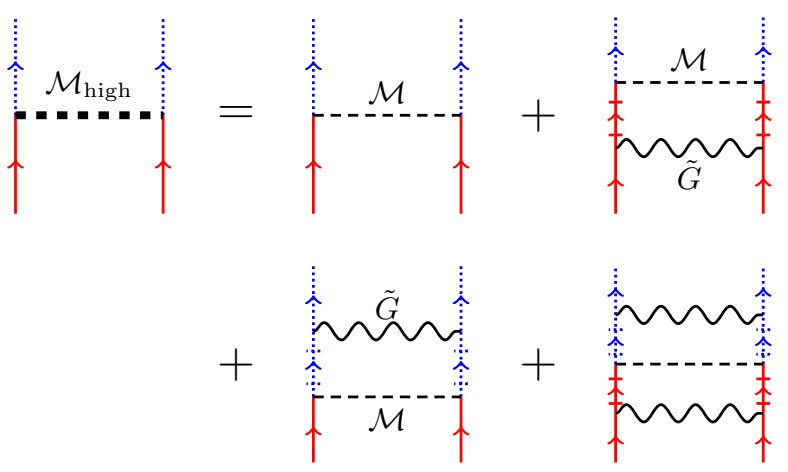

FIG. 3: (color online) An effective double-beta operator that accounts for short-range correlations in the nuclear wave function. Solid lines (red online) are neutrons and dotted line (blue online) are protons. The symbol $\tilde{G}$ represents the extension of $G$ to the $Q$ space.

more diagrams). The Bonn-C nucleon-nucleon interaction [15] was the starting point for the ladder-diagrams that enter the $G$ matrix. After summing all diagrams through third order in $G$ and the folded diagrams based on those, the authors adjusted the interaction by fitting certain components to spectra.

To be as consistent as possible with the calculations of Ref. [10] in constructing our effective decay operator for ${ }^{82} \mathrm{Se}$, we use the same Bonn-C interaction, the same valence space $\left(f_{5 / 2} p g_{9 / 2}\right)$, the same oscillator parameter $\left(b=82^{1 / 6} \mathrm{MeV}\right)$, and the same average energy $(\bar{E}=$ $10.08 \mathrm{MeV}$ ) as that reference throughout. After calcu-

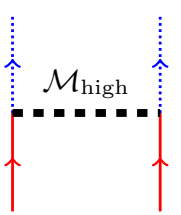

(a)

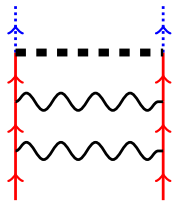

(d)

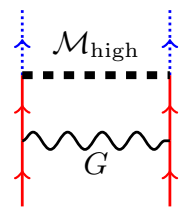

(b)

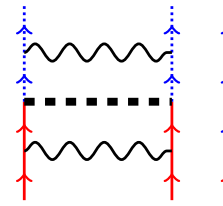

(e)

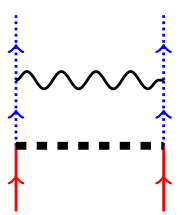

(c)

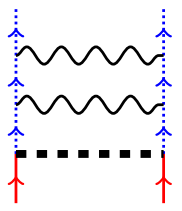

(f)

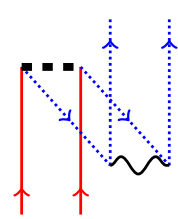

(g)

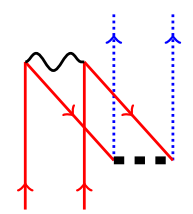

(h)

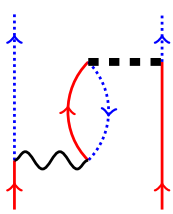

(i)

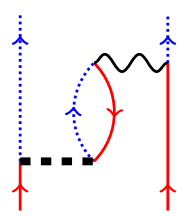

(j)
FIG. 4: (color online) Low order diagrams (in $G$ ) contributing to the final effective double-beta operator. The thick dashed line is $\mathcal{M}_{\text {high }}$, expressed diagrammatically in Fig. 2 
lating the $G$ matrix and its extension to the $Q$ space, we evaluate $\mathcal{M}_{\text {high }}$ in Fig. 3 and the more involved effectiveoperator diagrams in Fig. 4. Our only modification to the standard procedure is to prohibit intermediate particles in diagrams (i) and (j) from occupying levels that are essentially full in ${ }^{82} \mathrm{Se}$ and ${ }^{82} \mathrm{Kr}$; these Pauli-forbidden contributions, if included, would be canceled by higherorder diagrams that we do not evaluate here. Finally, we combine the matrix elements of the effective two-body operator (at several stages of approximation) with the 65 independent two-body ground-state-to-ground-state transition densities from the shell-model calculation of Ref. [10 to obtain a transition matrix element for the decay ${ }^{82} \mathrm{Se} \longrightarrow{ }^{82} \mathrm{Kr}$.

\section{RESULTS}

\section{A. High-energy states and short-range correlations}

Before looking at long-range corrections, we report the effects of the ladder diagrams shown in Fig. 3. The treatment of short-range correlations these diagrams represent is completely well defined; one knows exactly what it includes and what it omits, and there is no double counting. To look at the spatial structure of the correlations we define a two-body double-beta correlation function $C^{G T}(r)$ by making the substitution $H\left(r_{a b}, \bar{E}\right) \longrightarrow$ $H\left(r_{a b}, \bar{E}\right) \delta\left(r-r_{a b}\right)$ in the Gamow-Teller transition operator.

Figure 5 displays the results for the decay of ${ }^{82} \mathrm{Se}$ with the shell-model transition densities mentioned above, when the boundary between "high-energy" singleparticle states (ruled in the diagrams) and lower-energy states lies $4 \hbar \omega$ above the valence $f p$ shell. The results change only very slowly as the boundary is moved up from that point. The function in the top panel, labeled with the subscript 0 , corresponds to the simple GT operator of Eq. (5); the function in the bottom panel includes modifications to that operator from the weak nucleon form factors and higher-order terms in the weak current (see, e.g., Refs. 3] and [16] for definitions).

Both panels show the suppression of short-range contributions by the ladders, though the suppression is weaker in the lower panel because the nucleon form factors cut out some short-distance neutrino exchange by themselves. But in neither case is the matrix element reduced very much by the correlations: for the simple operator the reduction is about $8 \%$, and for the full operator it is less than $3 \%$. The reason, as the figure shows, is that probability density is shifted from very short distances to around $r=1 \mathrm{fm}$, where the function $H(r, \bar{E})$ is still large.

This behavior is similar to that found in Ref. [16], which constructs correlated wave functions by solving coupled-cluster equations at the one and two-body level. There are some differences between that treatment of short-range effects and ours: the coupled-clusters method

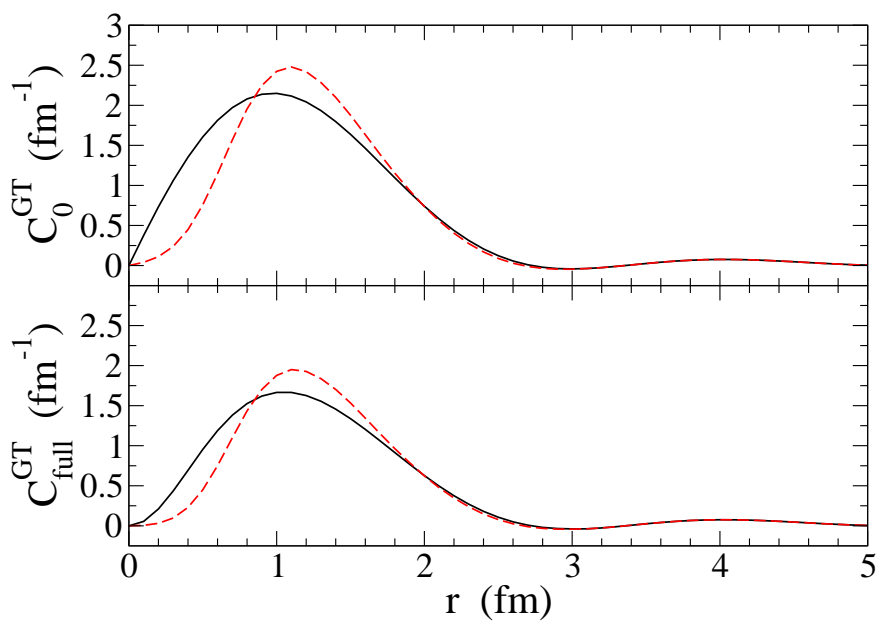

FIG. 5: (color online) The radial distribution $C^{G T}(r)$ that, when integrated, produces the Gamow-Teller $0 \nu$ matrix element for the decay of ${ }^{82} \mathrm{Se}$. The top panel corresponds to the simple operator given in Eq. (5) and the bottom panel includes momentum-transfer dependent form factors and forbidden operators (see text). The solid lines correspond to the bare operator and the dashed lines to the effective operator generated by summing the high-energy ladders in Fig. 4.

includes Hartree-Fock-like effects, sums hole-hole ladders as well as particle-particle ladders, and has no double partitioning. Nevertheless, both methods include much of the same physics and should yield similar results. To test the similarity we repeated our calculation with the Argonne V18 interaction used if Ref. [16 in place of the Bonn-C interaction. Figure 6 shows the results for the simple-operator GT correlation function discussed above along with the corresponding coupled cluster re-

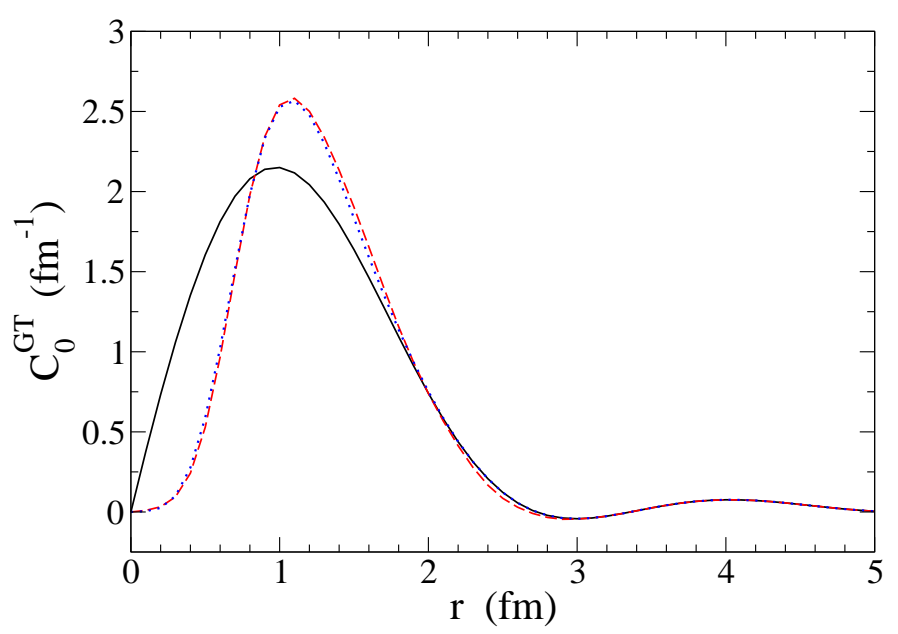

FIG. 6: (color online) Same as the top panel of Fig. 5 but with the dashed line from a calculation with the Argonne V18 potential in place of Bonn-C. The dotted line, nearly indistinguishable from the dashed line, comes from a fit to coupled-cluster results [16] with the Argonne potential. 
TABLE I: Renormalization of $M_{G T}^{0 \nu}$ (from equation (5)) for the decay ${ }^{82} \mathrm{Se} \longrightarrow{ }^{82} \mathrm{Kr}$.

\begin{tabular}{c|ccccc} 
boundary & bare & (a) & (a)-(f) & $(\mathrm{a})-(\mathrm{h})$ & all \\
\hline $4 \hbar \omega$ & 3.33 & 3.07 & 4.15 & 5.38 & 3.05 \\
$5 \hbar \omega$ & 3.33 & 3.06 & 4.17 & 5.39 & 3.15 \\
$6 \hbar \omega$ & 3.33 & 3.05 & 4.16 & 5.39 & 3.21 \\
$7 \hbar \omega$ & 3.33 & 3.06 & 4.17 & 5.39 & 3.28 \\
$8 \hbar \omega$ & 3.33 & 3.06 & 4.17 & 5.39 & 3.35
\end{tabular}

sults, which we generated by using the phenomenological Jastrow-function fit reported in Ref. [16. The two correlation functions are almost indistinguishable. These results, alongside those of Ref. [8, 9, mean that the phenomenological Jastrow function from Ref. 7], used reflexively for a long time, almost certainly overestimates the quenching due to short-range correlations.

\section{B. Longer-range correlations}

When the correlations induced by the diagrams in Fig. 4 are added to the short-range effects discussed above, the matrix element changes further. Results for the GamowTeller part of the matrix element (equation (5), without form factors or forbidden currents) appear in table I]

The table successively adds the results from three classes of diagrams: the high-energy ladders discussed above (labeled (a) in Fig. 4), the diagrams with the highenergy ladders embedded in lower energy ladders (labeled (b) - (f)), the 4-particle 2-hole diagrams (g) and (h), and finally the core-polarization diagrams (i) and (j). Each row corresponds to a different boundary (measured in $\hbar \omega$ from the $f p$ shell) between the "high-energy" particle levels, denoted by ruled lines in the figures, and the lower-energy levels. All the diagrams except the last two in Fig. 4 are insensitive to this boundary if it is above about $4 \hbar \omega$. The core polarization graphs, by contrast still have not converged at 8 (or even 9) $\hbar \omega$. We are unable to carry the calculation beyond that point. Corepolarization graphs in the effective interaction are notorious for converging very slowly, sometimes taking 20 or more $\hbar \omega$ [17.

The table shows several things. First, the short-range correlations, as discussed previously, damp the bare matrix element by about $8 \%$. Second, the ladder and $4-$ particle-2-hole contributions then increase the matrix element by about $75 \%$. These graphs contain pairing matrix elements that promote particles into unoccupied levels, unblocking the transition. Finally, the core-polarization diagrams decrease the matrix element so that after summing particle-hole configurations up to $8 \hbar \omega$ our matrix element is only marginally bigger than the bare version.
The core-polarization graphs contain the neutron-proton interaction, the correlations from which are known to counteract the effects of pairing. Cutting off the sum at $8 \hbar \omega$, however, probably exaggerates the size of this counteraction; the bulk of the effect comes from low energy levels, and contributions from higher-energy levels actually increase the matrix element again. Thus, our full result (in the column labeled "all") grows with the boundary between low and high-energy states, and is still growing at our maximum value.

All these statements remain true when true when we include the Fermi term in Eq. (4) - resulting in a total $M_{0 \nu}$ of 3.95 at $8 \hbar \omega$ vs. the bare value 3.78 - or add the effects of form factors. We have not included forbidden currents in the full calculations, but do not expect them to change the pattern reported in the table.

As just noted, the effects of the pairing and neutronproton correlations cancel each other to a significant extent. The cancellation resembles what happens in the QRPA, which includes a portion of the effects calculated here through the use of a relatively large single-particle space. When all is said and done, our result, is a bit larger (and continuing to grow at $8 \hbar \omega$ ) than result at the bottom of column (a). That number is the bare matrix element corrected for short-range correlations, like the results reported in Refs. [2] and [10. The use of our effective operator thus improves the agreement between the shell model and QRPA, though the convergence issues keep us from saying by exactly how much. One would expect something similar for ${ }^{76} \mathrm{Ge}$, which has only six fewer nucleons and is treated in the same model space.

Of course, the large corrections from individual graphs mean that higher order corrections may be sizeable as well. One cannot, therefore, take the results of this firstorder estimate too seriously. In particular, it would not be correct to conclude that the near cancellation between pairing and neutron-proton correlations persists to higher order and/or when many-body diagrams are included. The large individual components indicate that the contributions of states outside the model space are significant and may or may not cancel one another in a better calculation. A more reliable nonperturbative evaluation of the corrections is very important, and we are working in that direction.

\section{Acknowledgments}

We acknowledge useful discussions with Morten Hjorth-Jensen and thank Alfredo Poves for supplying shell-model densities. This work was supported in part by the U.S. Department of Energy under Contract No. DE-FG02-97ER41019 with UNC and Contract No. DEAC05-00OR22725 with UT-Battelle, LLC.
[1] F. T. Avignone III, S. R. Elliott, and J. Engel, Rev. Mod. Phys. 80, 481 (2008).
[2] J. Menendez, A. Poves, E. Caurier, and F. Nowacki, Nucl. 
Phys. A 818, 139 (2009).

[3] F. Šimkovic, A. Faessler, V. Rodin, P. Vogel, and J. Engel, Phys. Rev. C 77, 045503 (2008).

[4] P. J. Ellis and E. Osnes, Rev. Mod. Phys. 49, 777 (1977).

[5] M. Hjorth-Jensen, T. T. S. Kuo, and E. Osnes, Phys. Rep. 261, 125 (1995).

[6] E. Caurier, G. Martnez-Pinedo, F. Nowacki, A. Poves, and A. P. Zuker, Rev. Mod. Phys. 77, 427 (2005).

[7] G. Miller and J. Spencer, Ann. Phys. 100, 562 (1976).

[8] M. Kortelainen, O. Civitarese, J. Suhonen, and J. Toivanen, Phys. Lett. B 647, 128 (2007).

[9] M. Kortelainen and J. Suhonen, Phs. Rev. C 75, 051303 (2007).

[10] E. Caurier, J. Menendez, F. Nowacki, and A. Poves,
Phys. Rev. Lett. 100, 052503 (2008).

[11] B. Brandow, Rev. Mod. Phys. 39, 771 (1967).

[12] C. Bloch and J. Horowitz, Nucl. Phys. 8, 91 (1958).

[13] L. Coraggio, A. Covello, A. Gargano, N. Itaco, and T. T. S. Kuo, Prog. Part. Nucl. Phys. 62, 135 (2009).

[14] M. Dufour and A. P. Zuker, Phys. Rev. C 54, 1641 (1996).

[15] R. Machleidt, Adv. Nucl. Phys. 19, 189 (1989).

[16] F. Šimkovic, A. Faessler, H. Müther, V. Rodin, and M. Staut, arXiv:0902.0331 (2009).

[17] J. Vary, P. D. Sauer, and C. W. Wong, Phys. Rev. C 7, 1776 (1973). 